\title{
Diabéticos fumadores: uma população de elevado risco que muito beneficia em deixar de fumar
}

Luís Rebelo ${ }^{1}$

\section{RESUMO}

A relação entre a diabetes e o tabagismo é há muito conhecida. Estima-se que um em cada cinco diabético seja fumador, acrescentando ao risco cardiovascular base da sua doença também este por definição evitável. Por outro lado, os fumadores têm também maior risco de vir a apresentar diabetes.

É fundamental levar as pessoas com diabetes a não fumar ou se o fizerem a abandonar o hábito. Este grupo deve ser prioritário nos programas de cessação tabágica.

Palavras-chave: Diabetes Mellitus tipo 2, Tabagismo; Cessação tabágica; Prevenção.

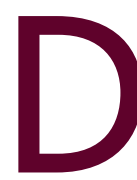
e acordo com Roderick e colaboradores, ${ }^{1}$ com base numa revisão sistemática realizada, concluiu-se que um em cada cinco doentes diabéticos tipo 2 fuma. Foi uma análise global que incluiu 74 estudos e 3,2 milhões de participantes de 33 países.

Em Portugal não se conhecem dados sobre a prevalência dos fumadores com diabetes mellitus tipo 2. Mas conhecem-se as prevalências quer de fumadores quer de diabéticos, ambas elevadas.

Segundo dados publicados pelo Observatório Nacional da Diabetes em $2016^{2}$ e referentes a 2015, a prevalência estimada da diabetes na população portuguesa com idades compreendidas entre os 20 e os 79 anos (7,7 milhões de indivíduos) foi de 13,3\%, isto é, mais de um milhão de portugueses neste grupo etário tem diabetes.

Quanto ao consumo do tabaco, o Instituto Nacional de Estatística divulgou os principais resultados do Inquérito Nacional de Saúde 2019, realizado em todo o

1. Médico de família. Responsável do Programa de Prevenção e Controlo do Tabagismo da ARSLVT. Lisboa, Portugal. Responsável pela Consulta de Cessação Tabágica da USF do Parque. Lisboa, Portugal. Professor Auxiliar. Faculdade de Medicina da Universidade de Lisboa. Lisboa, Portugal. território nacional, entre setembro de 2019 e janeiro de 2020. Em 2019, 17,0\% da população com 15 ou mais anos era fumadora, 1,3 milhões de pessoas $(14,2 \%)$ fumavam diariamente e 248 mil (2,8\%) faziam-no ocasionalmente. $^{3}$

\section{MORTALIDADE ENTRE FUMADORES POR DIABETES EM PORTUGAL}

A Direção-Geral da Saúde, no seu relatório de 2019 sobre a prevenção e controlo do tabagismo, escreve que em 2017, de acordo com estimativas elaboradas pelo Institute for Health Metrics and Evaluation, morreram em Portugal 13.104 pessoas por doenças atribuíveis ao tabaco. Destas, 10.588 homens e 2.515 mulheres. Por diabetes mellitus tipo 2 morreram $334(9,8 \%)$, das quais $201(13,5 \%)$ eram do sexo masculino e $133(6,9 \%)$ do sexo feminino. Em 2017, a diabetes mellitus contribuiu com $3,8 \%$ do total de óbitos ocorridos. ${ }^{4}$

\section{MORTALIDADE POR DOENÇA CARDIOVASCULAR EM FUMADORES DIABÉTICOS}

Pan e colaboradores ${ }^{5}$ realizaram uma revisão sistemática e uma meta-análise de estudos de coorte prospetivos em que avaliaram a relação entre o consumo de 
tabaco por fumadores ativos e a mortalidade e ocorrência de eventos cardiovasculares em doentes diabéticos. Concluíram que o consumo de tabaco está significativamente associado a um risco elevado de mortalidade total e cardiovascular entre doentes diabéticos cerca de $50 \%$ de excesso de mortalidade. Os ex-fumadores ainda mantêm um risco superior aos diabéticos que nunca fumaram. A cessação tabágica reduz para cerca de $19 \%$ esse risco em comparação com os que continuaram a fumar - resultados que sugerem o benefício da cessação tabágica nestes doentes.

São ainda Pan e seus colaboradores ${ }^{6}$ que concluíram que o risco também existe, embora com menor expressão, para os diabéticos expostos ao fumo ambiental.

\section{CONSUMO DE TABACO E DIABETES}

$\mathrm{O}$ Centers for Disease Control and Prevention no seu relatório de 2014, THE HEALTH CONSEQUENCES OF SMOKING - 50 YEARS OF PROGRESS, ${ }^{7}$ inclui pela primeira vez a diabetes mellitus tipo 2 como um dos efeitos causais do consumo do tabaco. São extraídas três conclusões:

1. A evidência é suficiente para inferir que o consumo de tabaco é causa de diabetes;

2. O risco de desenvolver diabetes é 30 a $40 \%$ superior entre fumadores ativos em comparação com não fumadores;

3. Existe uma relação dose/resposta positiva entre o número de cigarros fumados e o risco de desenvolver diabetes.

\section{MECANISMO DE AÇÃO}

Purcell e colaboradores ${ }^{8}$ afirmam que os mecanismos biológicos plausíveis de explicar a associação consumo de tabaco e diabetes incluem o aumento da obesidade central em fumadores, aumento da inflamação e stress oxidativo, aumento da resistência à insulina, secreção de insulina alterada e outras alterações da função pancreática observadas em fumadores. Também é possível que fatores genéticos desempenhem um papel na associação entre o início do tabagismo e a diabetes tipo 2.

\section{METABOLISMO DA NICOTINA EM DOENTES DIABÉTICOS}

O doente diabético tipo 2 metaboliza a nicotina mais rapidamente que o fumador não diabético, o que pode facilitar a dependência à nicotina. Nos fumadores com uma rápida metabolização, diabéticos ou não, o consumo é normalmente superior em número de cigarros e o consequente dano também. Sabe-se que o metabolismo da nicotina varia de fumador para fumador e alguns dos fatores que interferem são o sexo, a raça, o índice de massa corporal e a intensidade no fumar. ${ }^{9}$

\section{TABAGISMO, CESSAÇÃO TABÁGICA E INTERAÇÕES MEDICAMENTOSAS}

Os químicos do fumo do tabaco interagem com os medicamentos, alterando a sua farmacocinética e a sua farmacodinâmica. Exemplo da farmacocinética alterada passa-se com os antipsicóticos e os antidepressivos, em que os químicos do fumo do tabaco que estão em causa são sobretudo os hidrocarbonetos policíclicos aromáticos. Exemplo da farmacodinâmica alterada encontra-se no caso da metadona ou das benzodiazepinas e é a nicotina que tem um papel relevante.

A interação medicamentosa pode variar com o início do consumo, a paragem ou o número de cigarros fumados.

A maioria das interações medicamentosas é causada por substâncias não nicotínicas com ação hepática, via citocromo P 450 (enzimas CYP 1A2 e CYP 2B6), afetando a absorção, distribuição, metabolização ou eliminação da medicação). ${ }^{10-11}$

\section{CESSAÇÃO TABÁGICA EM DIABÉTICOS}

Purcell e colaboradores ${ }^{8}$ afirmam que o risco que o fumador diabético apresenta existe e vai manter-se mesmo depois de ter deixado de fumar. Pode mesmo aumentar nos três primeiros anos, só diminuindo após os quatro, cinco anos. Somente ao fim de dez anos se estima que o risco seja semelhante ao dos diabéticos não fumadores.

Sabe-se que ao deixar de fumar, e durante dois ou três anos, o diabético terá mais dificuldade em controlar a sua diabetes. É necessário considerar que o fumador, ao deixar de fumar, pode aumentar de peso e vir a tornar aparente uma diabetes inaugural, sobretudo nos casos em que já existia excesso ponderal.

Altas doses de nicotina interferem negativamente na ação da insulina, pelo que estes doentes vão necessitar de doses mais elevadas. Após oito semanas sem fumar, 
a insulina começa a recuperar progressivamente a sua efetividade. Com a cessação tabágica, os doentes diabéticos vão necessitar de menores doses de insulina e possivelmente de antidiabéticos orais.

No que se refere à medicação sabe-se que o bupropiom está contraindicado e, se for usado, deve ser com cuidado devido à hipótese de ocorrência de convulsões. A vareniclina não tem interações medicamentosas conhecidas, pelo que está indicada. Os substitutos de nicotina podem ser usados com as limitações conhecidas. As combinações terapêuticas devem ser usadas com reserva, pois potenciam os efeitos secundários descritos.

A pouca adesão dos doentes diabéticos aos programas de cessação tabágica é um facto e foi estudada.Vários fatores podem estar presentes - menor satisfação com o seu estado de saúde, iliteracia sobre o consumo de tabaco e diabetes, desconhecimento dos resultados dos programas de cessação tabágica e presença de mais sentimentos negativos.

\section{EM CONCLUSÃO}

Estima-se que no mundo um em cada cinco diabéticos fume. Na Administração Regional de Saúde de Lisboa eVale do Tejo, em outubro de 2019, um em cada dez diabéticos estava registado como fumador ativo.

Em Portugal estima-se que, no ano de 2017, tenham morrido $334(9,8 \%)$ diabéticos devido ao consumo do tabaco. Um em cada cinco mortes por diabetes tipo 2 foi observada em homens fumadores dos 50 aos 69 anos.

O risco de desenvolver diabetes é 30 a $40 \%$ superior entre fumadores ativos em comparação com não fumadores.

Os diabéticos que fumam têm um risco acrescido de cerca de $50 \%$ de morrerem por doença cardiovascular em comparação com os não fumadores.

Os fumadores diabéticos devem ser considerados prioritários nos programas de cessação tabágica. É essencial que deixem de fumar para limitarem o desenvolvimento das complicações micro e macrovasculares da diabetes.

Recomenda-se fortemente que se reduza a experimentação de tabaco entre os jovens, pois será uma medida preventiva da ocorrência da diabetes tipo $2 \mathrm{em}$ idade adulta.
O risco de falta de controlo metabólico é superior nos três a cinco anos após ter deixado de fumar em comparação com os diabéticos que se mantêm a fumar.

É necessário estar mais atento ao controlo metabólico da diabetes durante todo o programa de cessação tabágica.

Evitar medicar com bupropiom os diabéticos fumadores e rever a terapêutica diabética e não diabética pós cessação tabágica.

\section{REFERÊNCIAS BIBLIOGRÁFICAS}

1. Roderick P, Turner V, Readshaw A, Dogar O, Siddiqi K. The global prevalence of tobacco use in type 2 diabetes mellitus patients: a systematic review and meta-analysis. Diabetes Res Clin Pract. 2019;154:52-65.

2. Observatório da Diabetes. Diabetes: factos e números - o ano de 2015 (relatório anual do Observatório Nacional da Diabetes) [Internet]. Lisboa: Sociedade Portuguesa de Diabetologia; 2016. Available from: https://www.sns.gov.pt/wp-content/uploads/2017/03/OND-2017_ Anexo2.pdf

3. Instituto Nacional de Estatística. Inquérito nacional de saúde 2019. Lisboa: INE; 2020.

4. Direção-Geral da Saúde. Programa nacional para a prevenção e controlo do tabagismo [Internet]. Lisboa: DGS; 2019. Available from: https://www.sns.gov.pt/wp-content/uploads/2019/11/RelatorioTabaco2019.pdf.pdf

5. Pan A, Wang Y, Talaei M, Hu FB. Relation of smoking with total mortality and cardiovascular events among patients with diabetes mellitus: a meta-analysis and systematic review. Circulation. 2015;132(19): 1795-804.

6. Pan A, Wang Y, Talaei M, Hu FB, WT. Relation of active, passive, and quitting smoking with incident type 2 diabetes: a systematic review and meta-analysis. Lancet Diabetes Endocrinol. 2015;3(12):958-67.

7. U.S. Department of Health and Human Services. The health consequences of smoking - 50 years of progress: a report of the Surgeon General [Internet]. Atlanta: Centers for Disease Control and Prevention, National Center for Chronic Disease Prevention and Health Promotion, Office on Smoking and Health, 2014. Available from: https://www.ncbi. nlm.nih.gov/books/NBK179276/

8. Purcell K, Greenhalgh EM, Winstanley MH. Smoking and diabetes. In: Scollo MM, Winstanley MH, editors. Tobacco in Australia: facts and issues. Melbourne: Cancer Council Victoria; 2020. chapter 3.16.

9. Keith RJ, Riggs DW, Conklin DJ, Lorkiewicz P, Srivastava S, Bhatnagar A, et al. Nicotine metabolism in adults with type 2 diabetes. Nicotine Tob Res. 2019;21(6):846-9.

10. Hunter New England Area Health Service. Drug interactions with smoking. Sidney: HNEAHS; 2008.

11. Lucas $C$, Martin J. Smoking and drug interactions. Aust Prescr. 2013;36:102-4.

\section{CONFLITO DE INTERESSES}

O autor declara não possuir quaisquer conflitos de interesse. 
ENDEREÇO PARA CORRESPONDÊNCIA

Luís Rebelo

E-mail: Irebelo@sapo.pt

https://orcid.org/0000-0002-2508-2036
Recebido em 29-01-2021

Aceite para publicação em 07-03-2021

\section{ABSTRACT}

\section{PEOPLE LIVING WITH DIABETES WHO SMOKE: A HIGH-RISK POPULATION THAT MOST BENEFITS FROM QUIT}

\section{SMOKING}

The relation between diabetes and smoking is well known for long. About one in five people living with diabetes is a smoker, adding to their basal cardiovascular risk. Smoking risk is by definition preventable. On the other hand, smokers are also at greater risk of developing diabetes.

It is crucial to prevent people to start smoking, and in the diabetics who smoke to help them to stop. This group should be a priority in smoking cessation programs.

Keywords: Type 2 Diabetes Mellitus, Smoking, Smoking cessation, Prevention. 